\title{
Retrospective analysis of 19 papulopustular rosacea cases treated with oral minocycline and supramolecular salicylic acid $30 \%$ chemical peels
}

\author{
LIAN WANG ${ }^{*}$, XIAO-HUA LI ${ }^{*}$, XIANG WEN, XIAO-XUE LI, DAN DU, YONG LI and XIAN JIANG \\ Department of Dermatology, West China Hospital, Sichuan University, Chengdu, Sichuan 610041, P.R. China
}

Received June 24, 2019; Accepted March 19, 2020

DOI: $10.3892 /$ etm. 2020.8740

\begin{abstract}
Papulopustular rosacea (PPR) is characterized by central facial erythema and transient papules and/or pustules, with or without telangiectases. The treatment of PPR is challenging due to the unclear and complex pathogenesis. In the present retrospective study, patients with PPR treated with oral minocycline and supramolecular salicylic acid (SSA) 30\% chemical peels enrolled between June 2018 and June 2019 were evaluated. All patients were treated with $50 \mathrm{mg}$ minocycline twice a day and SSA 30\% twice a month. A total of 19 patients were enrolled and all received the therapy for 12 weeks. A significant reduction of rosacea severity was observed by Investigator Severity Assessment (ISA) after treatment; the mean score reduced from $3.32 \pm 0.6$ at baseline to $0.89 \pm 0.7(\mathrm{P}<0.01)$ at 12 weeks. After 12 weeks, all patients achieved at least a 'moderate response' and 17 patients (89.47\%) obtained 'excellent improvement' in the Investigator Global Assessment of efficacy. No obvious adverse reactions were observed during each patient's visit. In conclusion, the combination treatment of minocycline and SSA 30\% was an effective therapy for PPR. The limitation of the present study was that it was a retrospective analysis; more high-quality, prospective, blinded, controlled clinical trials are required to evaluate the efficacy based on the current study.
\end{abstract}

\section{Introduction}

Rosacea is an inflammatory disorder associated with symptoms such as flushing, erythema, telangiectasia, pustules, papules and fibrosis affecting the central face (1). According to the National Rosacea Society, there are four rosacea

Correspondence to: Dr Xian Jiang, Department of Dermatology, West China Hospital, Sichuan University, 37 Guoxue Alley, Wuhou, Chengdu, Sichuan 610041, P.R. China

E-mail: jennyxianj@163.com

${ }^{*}$ Contributed equally

Key words: rosacea, papulopustular, salicylic acid, minocycline, chemical peels subtypes, namely, erythematotelangiectatic rosacea (ETR), papulopustular rosacea (PPR), phymatous rosacea $(\mathrm{PhR})$ and ocular rosacea (OR) (2). ETR, characterized by transient flushing and persistent centrofacial erythema with the presence or absence of telangiectasia, is the most common subtype, followed by PPR, which is characterized by central facial erythema, transient papules and/or pustules, with or without telangiectases (2). PhR is characterized by thickened skin with irregular surface nodularities, while OR is accompanied by characteristic ophthalmic symptoms (3). Therapy should be based on the patients' individual symptoms and subtypes. For ETR, brimonidine is approved for symptomatic relief of the erythema and lasers and light devices are effective in the treatment of telangiectasia and erythema (4). PhR can be treated with $\mathrm{CO}_{2}$ laser and sharp blade excision (5). The treatments of OR include pharmaceutical agents, laser and light based therapies, and surgical interventions (6).

Various treatments have been used for the management of PPR, including oral doxycycline, minocycline, topical azelaic acid and ivermectin, all of which are reported to have anti-inflammatory properties $(1,7,8)$. Combination therapy for rosacea often yields better results than monotherapy. Oral antimicrobials/isotretinoin combined with topical agents are the mainstay of therapy for PPR (9). However, the treatment of PPR is challenging due to its recurrence. Twice monthly chemical peels with supramolecular salicylic acid (SSA) $30 \%$, the main ingredient of which is salicylic acid (SA), has anti-inflammatory properties and whitening effects, and has been widely used to treat acne, hyperpigmentation and other inflammatory dermatosis (10). Therefore, in the present study, a retrospective analysis of 19 cases of PPR was conducted to assess the efficacy and safety of oral minocycline and SSA $30 \%$ in the treatment of PPR.

\section{Materials and methods}

Patients. A retrospective study was conducted on 19 patients (16 females and 3 males; 27-53 years old) with PPR who had been treated with oral minocycline (100 mg/day) and SSA $30 \%$ chemical peels (twice a month) between June 2018 and June 2019 in the Department of Dermatology, West China Hospital, Sichuan University. This study was conducted in accordance with the ethical guidelines of the Declaration of Helsinki and 
approved by Medical Ethic and Human Research Committee of West China Hospital (approval no. 2017.163). Written informed consent was obtained from each patient. Patients were required to use moisturizer and avoid sunlight. The inclusion criteria were as follows: i) Patients who were diagnosed as PPR and developed papules or pustules with central facial erythema; ii) patients who were treated with the above combination treatment for 12 weeks and complete information about them was available; and iii) patients who were followed up for an additional 8 weeks after the end of treatment. The exclusion criteria applied to patients were as follows: i) Patients who had used any other topical treatments or oral agents and/or chemical peel within the previous 4 weeks; ii) patients who were pregnant or breastfeeding; iii) patients who had a history of photo allergy, or tetracycline or SA allergy; iv) patients who had active facial herpes simplex or warts; v) patients with scar diathesis; and vi) patients with severe defects of the heart, lung, liver or kidney.

Method of application of SSA $30 \%$. SSA $30 \%$ was used twice a month. The chemical peel was performed in the treatment room of a dermatological department. Prior to treatment, each patient washed her/his face and then lay on the treatment bed. According to the instructions of the manufacturer, an appropriate amount of SSA (7 g/box) was added to a small therapeutic bowl. SA was combined with Poloxamer 407 (a kind of solubilizer) as an emulsifiable paste via supramolecular technology (Broda, Shanghai Rui Zhi Medicine Technology Co., Ltd.; patent numbers US8865143 and EP2689774), with an initial concentration of 5-8\%. After adding water $(2 \mathrm{ml})$, SSA was released from Poloxamer 407 and reached a concentration of $30 \%$. After stirring, the chemical substance was applied to the skin lesions. Sites to avoid with SSA included the eyes, nasal cavities and lips. After $20 \mathrm{~min}$, the chemical substance was washed with water. Patients were required to apply moisturizer and sun protection.

Clinical assessment. Rosacea lesions were imaged by VISIA (Canfield Imaging Systems) monthly. Investigator Severity Assessment (ISA) of rosacea severity was scored using a 5-point scale (11): 0, clear (no erythema and no papules/pustules); 1, almost clear (very mild erythema and very few small papules/pustules); 2 , mild (mild erythema and few small papules/pustules); 3, moderate (moderate erythema and several small or large papules/pustules); 4, severe (severe erythema and numerous small and/or large papules/pustules) by three blinded dermatologists to evaluate the clinical outcomes. If the assessment results of three physicians varied, another two physicians were enrolled to re-evaluate the outcome. Meanwhile, Investigator Global Assessment (IGA) of efficacy was scored using a 6-point scale (11): -1, worsening; 0 , no response; 1 , mild response ( $<50 \%$ improvement); 2 , moderate (50-80\% improvement); 3 , excellent ( $>80 \%$ improvement); 4 , complete response/clear. Patients were asked to carry out self-assessment for rosacea symptoms after the last treatment, grading the improvement of rosacea as 1, good; 2, fair; 3, poor; and 4 , even worse.

Statistical analysis. All statistical analyses were conducted using Graph Prism 7. The data measurements are presented as mean \pm standard deviation. Statistical analyses were carried out by repeated measures one-way analysis of variance (ANOVA) test with post hoc Tukey's tests. $\mathrm{P}<0.05$ was considered to indicate a statistically significant difference.

\section{Results}

Cases and patient characteristics. A total of 19 patients (16 females and 3 males) diagnosed with PPR were enrolled in the study, and received the therapy for 12 weeks. All 19 patients showed persistent erythema and transient papules/pustules, and presented $\geq 1$ subjective symptom. The characteristics of the patients were in accord with the typical characteristics of patients with PPR (Table I).

Clinical assessment. At 4, 8 and 12 weeks, a significant reduction in rosacea severity was observed in the ISA; the mean score reduced from $3.32 \pm 0.6$ at baseline to $2.21 \pm 0.7$ at 4 weeks, $1.53 \pm 0.8$ at 8 weeks, and $0.89 \pm 0.7$ at 12 weeks $(\mathrm{P}<0.05$; Fig. 1). In the IGA assessment, after 4 weeks, 10 patients $(52.63 \%)$ exhibited a 'moderate' 'response', and after 8 weeks, 18 patients $(94.74 \%)$ showed at least a 'moderate' response. After 12 weeks of treatment, all patients exhibited at least a 'moderate' response, and there were 17 patients $(89.47 \%)$ with 'excellent' improvement (Table II). Representative photographs and redness area on VISIA of four patients are shown in Figs. 2 and 3. None of the patients were scored as 'no response' or 'worsening'. After the last treatment, 17 patients $(89.47 \%)$ reported the improvement of rosacea as 'good', 2 patients reported 'fair', and no patients reported 'poor' or 'even worse'. There were 3 patients who reported mild burning sensation during the first SA chemical peel, while no adverse reactions were observed for other patients during the visit. At 4- and 8-week follow-up visits, no patients reported a relapse or worsening.

\section{Discussion}

The precise pathogenesis of rosacea is complex and remains unclear, although it is currently thought to have a genetic background, with neurovascular dysfunction, disorganized innate and acquired immunity (12). Karpouzis et al (13) observed a statistically significant predominance of tachykinin receptor $3 \mathrm{rs} 3733631 \mathrm{G}$ allele in PPR, indicating it may predispose the evolution of the disease. Chang et al (14) suggested a role for antigen presentation by class II HLA in the etiology of rosacea by genome-wide association study (GWAS). Similarly, a GWAS by Aponte et al (15) reported that rosacea is associated with gene regions that are involved in an inflammatory component. In the development of PPR, innate immune mediators, including Toll-like receptor (TLR) and nucleotide-binding oligomerization domain (NOD)-like receptor, can be activated by extracellular pathogen- or damage-associated molecular patterns and are required for innate immune peptide cathelicidin and inflammasome activation, which causes inflammatory infiltration and induces the activation of adaptive immune cells, facilitating the development of inflammatory papules and pustules (12). Symptoms of PPR are bothersome to patients and have a negative effect on their quality of life (16). Triggers include microbes, 
Table I. Characteristics of the 19 patients.

\begin{tabular}{lc}
\hline Characteristics & Value, $\mathrm{n}(\%)$ \\
\hline Skin phototype & \\
III & $13(68.42)$ \\
IV & $6(31.58)$ \\
Affected areas & \\
Nose and paranasal area & $19(10)$ \\
Cheek & $14(73.68)$ \\
Forehead & $4(21.05)$ \\
Manifestation & \\
Flushing & $13(68.42)$ \\
Erythema & $19(100)$ \\
Papules and pustules & $19(100)$ \\
Telangiectasia & $8(42.11)$ \\
Subjective symptoms & $17(89.47)$ \\
Burning & $6(31.58)$ \\
Itching &
\end{tabular}

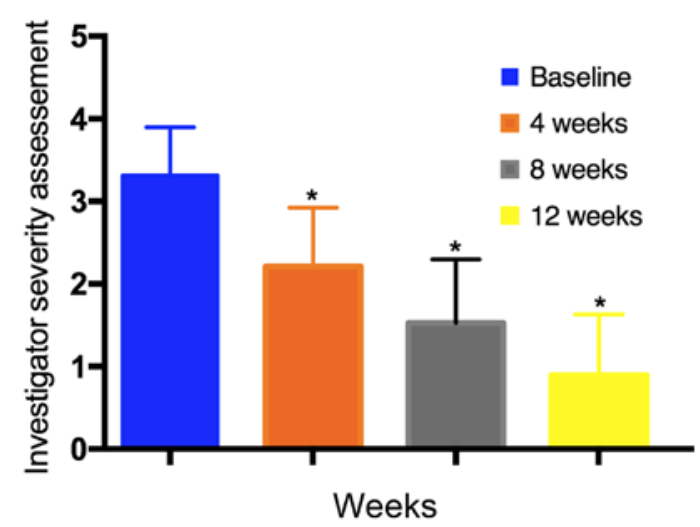

Figure 1 Changes in Investigator Severity Assessment at baseline, 4, 8 and 12 weeks. A statistically significant difference was detected among baseline and all the time points post-treatment. ${ }^{*} \mathrm{P}<0.05$.

ultraviolet radiation and stress, which can stimulate receptors such as TLR and NOD-like receptor. Dysregulated receptors induce the activation of neurogenic inflammation and innate immune pathways, such as the inflammasome activation and NF-kB pathways, resulting in abnormally sensitive skin and the production of distinct cytokines, chemokines, matrix metalloproteinases (MMPs) and prostanoids, which may facilitate the clinical manifestation of erythema, papules and pustules (12). However, treatment of PPR is difficult, particularly in patients with underlying persistent erythema (17). According to these mechanisms, the current therapeutics for PPR mainly include trigger avoidance, oral antimicrobials and topical agents to control inflammatory reactions (9). The present study reviewed the combination treatment of minocycline and SSA 30\% chemical peel for PPR in Department of Dermatology, West China Hospital, Sichuan University. The results of present study showed a gradual decrease in erythema and inflammatory lesions in 19 patients with PPR after receiving this treatment. Objective and subjective symptoms improved significantly without any relapse during the 8-week follow-up visit.

Despite the approval of doxycycline for the treatment of rosacea in 2006 by the USA Food and Drug Administration, subsequent studies have demonstrated that minocycline is effective and could be a good alternative treatment for those patients who, for any reason, are unable or unwilling to receive doxycycline $(8,18)$. A study by van der Linden et al (8) suggested that the efficacy of minocycline is comparable with that of doxycycline in treating PPR, and patients treated with minocycline group possessed a better quality of life compared with those treated with doxycycline. Minocycline, a type of broad-spectrum tetracycline, inhibits various pathways of inflammation. Its anti-inflammatory effect is attributed to the inhibition of MMPs, bacterial products that stimulate inflammation and phospholipase A2, and the suppression of neutrophil migration and chemotaxis, which play important roles in the pathogenesis of PPR $(8,12,19)$. Furthermore, minocycline can increase the hydration of the stratum corneum, which may facilitate the repair of the epidermal barrier function. Although uncommon, tetracycline can cause hyperpigmentation as a side effect, and there is an association between the duration of minocycline intake and the duration of pigmentation, with a median duration of 17 months (20). Therefore, when using minocycline, patients are required to avoid sunlight and long-term use.

SA at a $30 \%$ concentration is a naturally active ingredient with anti-inflammatory and keratin-exfoliating properties, which has been widely used in skin diseases, particularly in acne vulgaris (21). SA decreases sebum secretion through downregulation of the adenosine monophosphate-activated protein kinase/sterol regulatory element-binding protein-1 signaling pathway, and antagonizes the inflammatory response through inhibition of the $N F-\kappa B$ signaling pathway (22). In addition, SA at $20-30 \%$ concentration can disrupt intercorneocyte cohesion, causing a rapid differentiation of keratinocytes and peeling the entire epidermis, resulting in the reorganization of the epidermis and the removal of excess melanin $(23,24)$. Due to its antioxidant properties and inhibition of tyrosinase expression, SA can be used to treat pigmented dermatosis (25). However, SA is insoluble in water due to its lipophilic characteristics, and requires the addition of an alcoholic solution to be completely dissolved. With reversible and non-covalent bonding to form a water-soluble SSA complex, SSA 30\% is characterized by slow release upon application, and achieves its maximum efficacy at low $\mathrm{pH}$, reducing skin irritation (10).

Despite previous studies illustrating the treatment of rosacea with minocycline combined with other methods, such as pulsed dye laser and tranexamic acid, to the best of the authors' knowledge, there have been few studies written in English on the effectiveness of oral minocycline and chemical peel in rosacea or PPR $(18,26)$. Based on the advantages of minocycline and SSA, this combination modality was applied to treat PPR in the present study. Patients experienced a marked improvement in inflammatory lesions with regard to their clinical manifestations and redness area on VISIA. After 8 weeks, the majority 
Table II. Investigator Global Assessment of improvement of the 19 patients.

\begin{tabular}{lllllll}
\hline Treatment duration & Clear & Excellent & Moderate & Mild & No response & Worsening \\
\hline 4 weeks & $0(0.00)$ & $1(5.26)$ & $10(52.63)$ & $8(42.11)$ & $0(0.00)$ & $0(0.00)$ \\
8 weeks & $0(0.00)$ & $6(31.58)$ & $12(63.16)$ & $1(5.26)$ & $0(0.00)$ & $0(0.00)$ \\
12 weeks & $8(42.11)$ & $9(47.37)$ & $2(10.52)$ & $0(0.00)$ & $0(0.00)$ & $0(0.00)$ \\
\hline
\end{tabular}

Values are presented as $\mathrm{n}(\%)$.
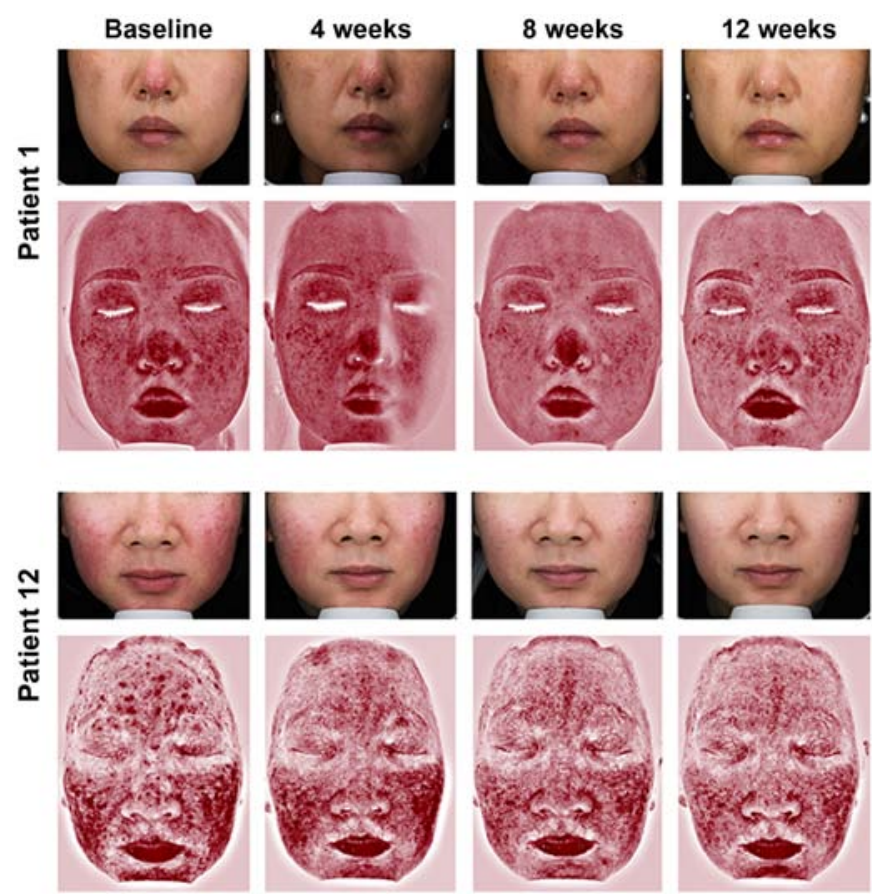

Figure 2. Clinical manifestations of patients 1 and 12 before and after 4,8 and 12 weeks of treatment with standard photographs (upper panels) and the redness area of VISIA (lower panels). Both patients achieved a 'complete response'.

of patients exhibited a moderate response. After the last treatment and follow-up for an additional 8 weeks, all patients experienced improvement and reported no obvious side effects. Although the exact mechanism by which SA acts in PPR is not clear, two factors are hypothesized to support its functional mechanism. First, SSA $30 \%$ can inhibit inflammation via the $\mathrm{NF}-\kappa \mathrm{B}$ signaling pathway and inhibit interleukin-1 $\beta$ and tumor necrosis factor involved in rosacea $(12,22)$. Second, the chemical exfoliation and whitening effect of SSA $30 \%$ facilitate the reorganization of the epidermis and prevent pigmentation.

There are certain limitations to the present study. First, the number of patients was limited. Second, it was a retrospective analysis with no control group; strict comparison between single and combination regimens should be performed for PPR. Third, further high-quality, prospective, blinded, controlled clinical trials are required to evaluate the efficacy of this retrospective study.

In conclusion, the present study demonstrates that combination treatment with minocycline and SSA $30 \%$ is effective for PPR. This retrospective study is of significance in guiding
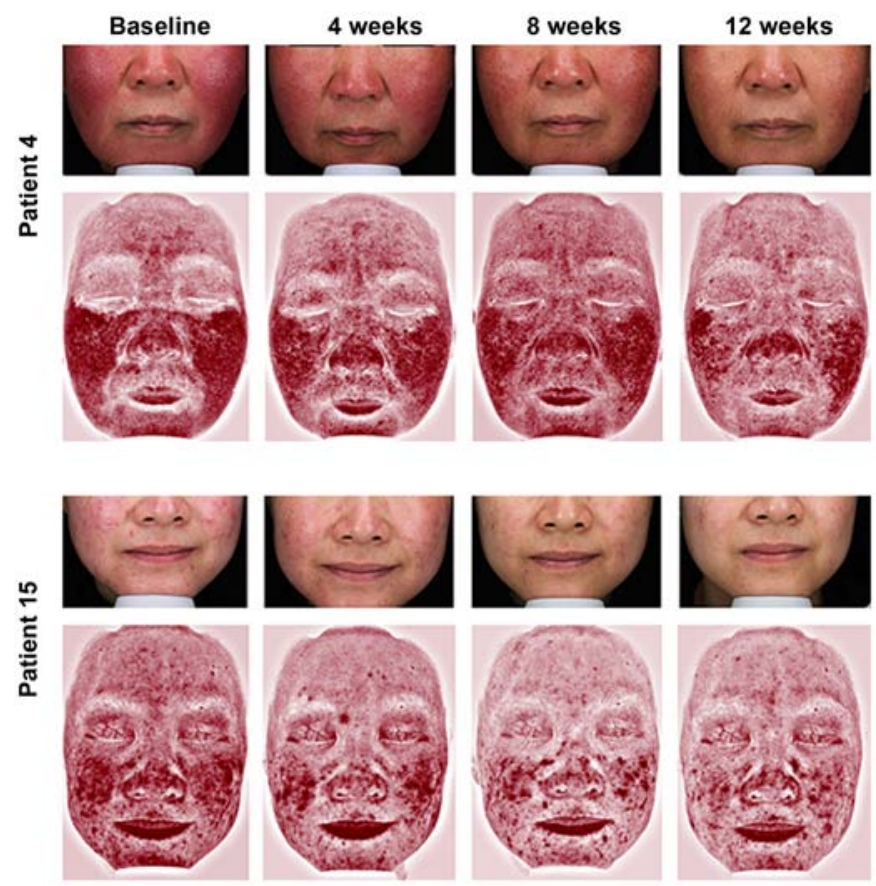

Figure 3. Clinical manifestations of patients 4 and 15 before and after 4 , 8 and 12 weeks of treatment with standard photographs (upper panels) and redness area of VISIA (lower panels). Both patients achieved an 'excellent response' (>80\% improvement).

clinical practice, and provides a new combination therapy that may be safe and effective in treating PPR.

\section{Acknowledgments}

The authors are grateful to Xiao-qin Xie (bachelor's degree) and Dan HAO (PhD) from Department of Dermatology, West China Hospital, Sichuan University for their comments on this paper and to Shanghai Rui Zhi Medicine Technology Co., Ltd., Shanghai, China for its technical support.

\section{Funding}

This work was financially supported by the transverse project of West China hospital (grant nos. HX-H1704048 and HX-H1902027).

\section{Availability of data and materials}

All data generated or analyzed during this study are included in this published article. 


\section{Authors' contributions}

LW, XW and XJ conceived and designed the study. LW, XHL, XXL, YL and XJ performed the experiments. LW and XW analyzed the data. DD assisted in the study design and discussed and interpreted the data. LW, XHL, and XJ wrote the manuscript. All authors read and approved the final manuscript.

\section{Ethics approval and consent to participate}

This study was conducted in accordance with the ethical guidelines of the Declaration of Helsinki and approved by Medical Ethics and Human Research Committee of West China Hospital (no. 2017.163).

\section{Patient consent for publication}

Written informed consent was obtained from each patient. All patients agreed to the publication of their photographs and their clinical data.

\section{Competing interests}

The authors declare that they have no competing interests.

\section{References}

1. Rainer BM, Kang S and Chien AL: Rosacea: Epidemiology, pathogenesis, and treatment. Dermatoendocrinol 9: e1361574, 2017.

2. Wilkin J, Dahl M, Detmar M, Drake L, Feinstein A, Odom R and Powell F: Standard classification of rosacea: Report of the national rosacea society expert committee on the classification and staging of rosacea. J Am Acad Dermatol 46: 584-587, 2002.

3. Two AM, Wu W, Gallo RL and Hata TR: Rosacea: Part I. Introduction, categorization, histology, pathogenesis, and risk factors. J Am Acad Dermatol 72: 749-760, 2015.

4. Piwnica D, Rosignoli C, de Menonville ST, Alvarez T, Schuppli Nollet M, Roye O, Jomard A and Aubert J: Vasoconstriction and anti-inflammatory properties of the selective $\alpha$-adrenergic receptor agonist brimonidine. J Dermatol Sci 75: 49-54, 2014.

5. Serowka KL, Saedi N, Dover JS and Zachary CB: Fractionated ablative carbon dioxide laser for the treatment of rhinophyma. Lasers Surg Med 46: 8-12, 2014.

6. Wladis EJ and Adam AP: Treatment of ocular rosacea. Surv Ophthalmol 63: 340-346, 2018.

7. Schaller M, Almeida LM, Bewley A, Cribier B, Dlova NC, Kautz G, Mannis M, Oon HH, Rajagopalan M, Steinhoff M, et al: Rosacea treatment update: Recommendations from the global ROSacea COnsensus (ROSCO) panel. Br J Dermatol 176 : 465-471, 2017.

8. van der Linden MMD, van Ratingen AR, van Rappard DC, Nieuwenburg SA and Spuls PI: DOMINO, doxycycline $40 \mathrm{mg}$ vs. minocycline $100 \mathrm{mg}$ in the treatment of rosacea: A randomized, single-blinded, noninferiority trial, comparing efficacy and safety. Br J Dermatol 176: 1465-1474, 2017.

9. McGregor SP, Alinia H, Snyder A, Tuchayi SM, Fleischer A Jr and Feldman SR: A review of the current modalities for the treatment of papulopustular rosacea. Dermatol Clin 36: 135-150, 2018.
10. Zheng Y, Yin S, Xia Y, Chen J, Ye C, Zeng Q and Lai W: Efficacy and safety of $2 \%$ supramolecular salicylic acid compared with $5 \%$ benzoyl peroxide $/ 0.1 \%$ adapalene in the acne treatment: A randomized, split-face, open-label, single-center study. Cutan Ocul Toxicol 38: 48-54, 2019.

11. Dall'Oglio F, Lacarrubba F, Luca M, Boscaglia S and Micali G: Clinical and erythema-directed imaging evaluation of papulo-pustular rosacea with topical ivermectin: A 32 weeks duration study. J Dermatolog Treat 30: 703-707, 2019.

12. Holmes AD and Steinhoff M: Integrative concepts of rosacea pathophysiology, clinical presentation and new therapeutics. Exp Dermatol 26: 659-667, 2017.

13. Karpouzis A, Avgeridis P, Tripsianis G, Gatzidou E, Kourmouli N and Veletza S: Assessment of tachykinin receptor 3' gene polymorphism rs3733631 in rosacea. Int Sch Res Notices 2015: 469402, 2015.

14. Chang ALS, Raber I, Xu J, Li R, Spitale R, Chen J, Kiefer AK, Tian C, Eriksson NK, Hinds DA and Tung JY: Assessment of the genetic basis of rosacea by genome-wide association study. J Invest Dermatol 135: 1548-1555, 2015.

15. Aponte JL, Chiano MN, Yerges-Armstrong LM, Hinds DA, Tian C, Gupta A, Guo C, Fraser DJ, Freudenberg JM, Rajpal DK, et al: Assessment of rosacea symptom severity by genome-wide association study and expression analysis highlights immuno-inflammatory and skin pigmentation genes. Hum Mol Genet 27: 2762-2772, 2018.

16. Wu Y, Fu C, Zhang W, Li C and Zhang J: The dermatology life quality index (DLQI) and the hospital anxiety and depression (HADS) in Chinese rosacea patients. Psychol Health Med 23: 369-374, 2018.

17. Lee WJ, Lee YJ, Lee MH, Won $\mathrm{CH}$, Chang SE, Choi JH and Lee MW: Prognosis of 234 rosacea patients according to clinical subtype: The significance of central facial erythema in the prognosis of rosacea. J Dermatol 43: 526-531, 2016.

18. Kwon HJ, Suh JH, Ko EJ and Kim BJ: Combination treatment of propranolol, minocycline, and tranexamic acid for effective control of rosacea. Dermatol Ther 30, 2017.

19. Perret LJ and Tait CP: Non-antibiotic properties of tetracyclines and their clinical application in dermatology. Australas J Dermatol 55: 111-118, 2014.

20. Dwyer CM, Cuddihy AM, Kerr RE, Chapman RS and Allam BF: Skin pigmentation due to minocycline treatment of facial dermatoses. Br J Dermatol 129: 158-162, 1993.

21. Thuangtong $\mathrm{R}$, Tangjaturonrusamee $\mathrm{C}$, Rattanaumpawan $\mathrm{P}$ and Ditre CM: Comparison of salicylic acid 30\% peel and pneumatic broadband light in the treatment of mild to moderately severe facial acne vulgaris. Cutis 100: 43-48, 2017.

22. Lu J, Cong T, Wen X, Li X, Du D, He G and Jiang X: Salicylic acid treats acne vulgaris by suppressing AMPK/SREBP1 pathway in sebocytes. Exp Dermatol 28: 786-794, 2019.

23. Sgontzou T, Armyra K, Kouris A, Bokotas C and Kontochristopoulos G: Repeated salicylic acid peels for the treatment of hyperplastic sebaceous glands in hypohidrotic ectodermal dysplasia. J Cosmet Laser Ther 16: 293-295, 2014.

24. Monheit GD and Chastain MA: Chemical peels. Facial Plast Surg Clin North Am 9: 239-255, viii, 2001.

25. Amann R and Peskar BA: Anti-inflammatory effects of aspirin and sodium salicylate. Eur J Pharmacol 447: 1-9, 2002.

26. Ko HS, Suh YJ, Byun JW, Choi GS and Shin J: Pulsed dye laser treatment combined with oral minocycline reduces recurrence rate of rosacea. Ann Dermatol 29: 543-547, 2017.

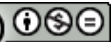

This work is licensed under a Creative Commons Attribution-NonCommercial-NoDerivatives 4.0 International (CC BY-NC-ND 4.0) License. 